\title{
ANALYSIS OF A DISEASE TRANSMISSION MODEL WITH TWO GROUPS OF INFECTIVES*
}

\author{
M. R. RAZVAN ${ }^{\dagger}$
}

\begin{abstract}
In this paper, we give a complete analysis of an SIS epidemiological model in a population of varying size with two dissimilar groups of infective individuals. It is mainly based on the discussion of the existence and stability of equilibria of the proportions system and the result is in terms of a threshold parameter which governs the stability of the disease free equilibrium.
\end{abstract}

1. Introduction. The social mixing structure of a population or a group of interacting populations plays a crucial role in the dynamics of an epidemic process. In almost all attempts to combine epidemiological data with mathematical modelling, there has been a recognition of the need to consider the structure of social interactions among the individuals in the populations. (See [5, 20] and references therein.) Many authors have considered the multigroup models in which heterogenous subpopulations may participate in the epidemic process with different parameters [4]. For SIS type models, a rather complete analysis of existence and global stability of a nontrivial epidemic state has been carried out by Lajmanovich and Yorke [14]. In their work, the size of each subpopulation is assumed to be constant. This assumption became an important but limiting component in the modelling of disease transmission [9].

A famous example for these subpopulations is the core group, i.e. the highly sexually active subgroups [8]. It has become increasingly clear that the transmission within and among core subgroups is an important factor in the transmission of venereal diseases [9]. In order to consider the core group in an SIS epidemiological model, we divide the population into two or more subgroups each of them consists of susceptible and infective individuals. Among these subpopulations, those with higher contact rates can be viewed as core groups $[12,13]$. Another example for multigroup models in epidemiology is the differential infectivity model introduced in $[10,11]$. In most of infectious diseases, the infection level varies widely among individuals and this causes various levels of infectivity. In a differential infectivity model, the susceptible subpopulation is assumed to be homogeneous and the infective population is subdivided into groups according to their infectiveness.

In this paper we examine an SIS model of disease transmission in a population of varying size with a homogeneous susceptible group and two dissimilar groups of infective individuals. We assume that a given fraction of new infected individuals enter each of these two groups. This model is similar to the differential infectivity model, but different from the core group model for the homogeneity of susceptible individuals. In fact this model applies to another type of core group that is postinfection core group, i.e. individuals that become part of the core group after being infected. This hypothesis is relevant to the behavior of infected individuals in our population. An example for post-infection core group is those individuals who are careless about the other one's health. They are infected like the other people, but transmit the disease much more, hence they are members of core group after being infected. In the case of contagious and fatal diseases, another example of post-infection core group has been observed. After being infected by a fatal disease via a number of

\footnotetext{
*Received October 26, 2000; accepted for publication September 19, 2001.

$\dagger$ Institute for Studies in Theoretical Physics and Mathematics, P.O.Box 11395-5746, Tehran, Iran (razvan@ipm.ir).
} 
contacts, a very small group of infected individuals may deliberately spread the disease to others because of anger or a desire to retaliate. Thus the post-infection core group may consist of those who are superspreaders because of their personal characteristics or psychological motivations. We analyze our model without any assumption on the involved parameters so that it can be applied to different interpretations of the model.

First of all, in the next section, we introduce the model which is a three dimensional ordinary differential equation homogeneous of degree one. For such a system it is more appropriate to consider the proportions equations which gives a planar system. In Section 3, we state a result concerning the nonexistence of certain types of solutions $[1,2,3]$ and show that every solution of the proportions system in the feasibility region tends to an equilibrium point. This reduces our problem to the discussion of the existence and stability of equilibria of the proportions system. Finally in Section 4, we give a complete global analysis of the proportions system in terms of a threshold parameter which governs the stability of the disease free equilibrium.

2. The Model. In order to derive our model, we divide the population into three groups: susceptibles, $S$, and two groups of infectives, $I_{1}$ and $I_{2}$, hence the total size of the population is $N=S+I_{1}+I_{2}$. In our model, the incidence function is of proportionate mixing type $[7,17]$ and natural births and deaths are proportional to the class numbers with all newborns susceptibles. We also assume that the birth rate of susceptibles may be more than that of infectives. This is similar to the demographic assumption in $[15,16]$. The excess death due to the disease among infectives is also considered. We use the following parameters which are positive unless otherwise specified:

$b$ : per capita birth rate of susceptibles,

$b_{1}$ : per capita birth rate of infectives assumed to lie in $[0, b]$.,

$d$ : per capita disease free death rate,

$\varepsilon$ : excess per capita death rate of infectives,

$\lambda_{1}$ : effective per capita contact rate of $I_{1}$,

$\lambda_{2}$ : effective per capita contact rate of $I_{2}$,

$\gamma_{1}$ : per capita recovery rate of $I_{1}$,

$\gamma_{2}$ : per capita recovery rate of $I_{2}$.

Therefore the reproduction term is $b N+b_{1}\left(I_{1}+I_{2}\right)=b_{1} N+b_{2} S$ where $b_{2}=b-b_{1} \geq 0$ and the incidence function with group $I_{j}$ is $\frac{\lambda_{j} I_{j}}{N}, j=1,2$. Similar to the differential infectivity model $[10,11]$, we assume that the susceptible individuals who have been infected, enter the group $I_{1}$ and $I_{2}$ of proportions $p$ and $q$ respectively, hence $p+q=1$. In applications, we may assume that $\lambda_{2} \gg \lambda_{1}$ and $q \ll 1$. Then $I_{2}$ can be considered as the highly infected group in the differential infectivity model or the post-infection core group. But we analysis our system in the general case in order to see the effect of each parameter more clearly.

The above hypotheses lead to the following system of differential equations in $\mathbb{R}_{+}^{3}$, where "'" denotes the derivatives with respect to $t$, the time.

$$
\begin{cases}S^{\prime}=b_{1} N+\left(b_{2}-d\right) S+\gamma_{1} I_{1}+\gamma_{2} I_{2}-\lambda_{1} \frac{I_{1} S}{N}-\lambda_{2} \frac{I_{2} S}{N}, & (2-1) \\ I^{\prime}{ }_{1}=p\left(\lambda_{1} \frac{I_{1} S}{N}+\lambda_{2} \frac{I_{2} S}{N}\right)-\left(d+\varepsilon+\gamma_{1}\right) I_{1}, & (2-2) \\ I^{\prime}{ }_{2}=q\left(\lambda_{1} \frac{I_{1} S}{N}+\lambda_{2} \frac{I_{2} S}{N}\right)-\left(d+\varepsilon+\gamma_{2}\right) I_{2} . & (2-3)\end{cases}
$$

The total population equation is obtained by adding the above three equations:

$$
N^{\prime}=\left(b_{1}-d\right) N+b_{2} S-\varepsilon\left(I_{1}+I_{2}\right)
$$


The system (2-1)-(2-3) is homogeneous of degree one and the population equation is linear. For such a system, it is more appropriate to consider proportions. If we set $s=\frac{S}{N}, i_{1}=\frac{I_{1}}{N}$ and $i_{2}=\frac{I_{2}}{N}$, we arrive at the following system of equations:

$$
\begin{cases}s^{\prime}=b_{1}(1-s)+b_{2} s(1-s)+\gamma_{1} i_{1}+\gamma_{2} i_{2}+\left(\varepsilon-\lambda_{1}\right) i_{1} s+\left(\varepsilon-\lambda_{2}\right) i_{2} s, & (2-1)^{\prime} \\ i_{1}^{\prime}=p s\left(\lambda_{1} i_{1}+\lambda_{2} i_{2}\right)+\varepsilon i_{1}\left(i_{1}+i_{2}\right)-\left(b_{1}+\varepsilon+\gamma_{1}\right) i_{1}-b_{2} s i_{1}, & (2-2)^{\prime} \\ i_{2}^{\prime}=q s\left(\lambda_{1} i_{1}+\lambda_{2} i_{2}\right)+\varepsilon i_{2}\left(i_{1}+i_{2}\right)-\left(b_{1}+\varepsilon+\gamma_{2}\right) i_{2}-b_{2} s i_{2} . & (2-3)^{\prime}\end{cases}
$$

In order to determine the asymptotic behavior of the solutions of this system of equations, we need some concepts of ODE related to our system. Given an autonomous system of ordinary differential equations in $\mathbb{R}^{n}$,

$$
\frac{d x}{d t}=f(x),
$$

we will denote by x.t the value of the solution of this system at time $t$, that is $x$ initially. For $V \subseteq \mathbb{R}^{n}, J \subseteq \mathbb{R}$, we let $V . J=\{x . t: x \in V, t \in J\}$. The set $V$ is called positively invariant if $V \cdot \mathbb{R}^{+}=V$. For $Y \subseteq \mathbb{R}^{n}$ the $\omega$-limit (resp. the $\alpha$-limit) set of $Y$ is defined to be the maximal invariant set in the closure of $Y .[0, \infty)($ resp. $Y .(-\infty, 0])$. An orbit $\gamma$ is a heteroclinic orbit if $\lim _{t \rightarrow-\infty} \gamma(t)=x$ and $\lim _{t \rightarrow+\infty} \gamma(t)=y$ where $x$ and $y$ are rest points and it is called a homoclinic orbit when $x$ coincides with $y$. A closed curve connecting several equilibria whose segments between successive equilibria are heteroclinic orbits is called a phase polygon. By a sink we mean an equilibrium at which all the eigenvalues of the linearized system have negative real parts. Such a point is called a source if all of these eigenvalues have positive real parts. If some of these eigenvalues have positive real parts and the others negative real parts, then the equilibrium is called a saddle point and it is called nondegenerate if all of these eigenvalues are nonzero.

3. Some Basic Results. We start our analysis with some basic results about the system $(2-1)^{\prime}-(2-3)^{\prime}$. If we set $\sum=s+i_{1}+i_{2}$, then $\sum^{\prime}=\left(1-\sum\right)\left(b_{1}+b_{2} s-\right.$ $\left.\varepsilon i_{1}-\varepsilon i_{2}\right)$. Therefore the plane $\sum=1$ is invariant. We consider the feasibility region

$$
D=\left\{\left(s, i_{1}, i_{2}\right): s+i_{1}+i_{2}=1, s \geq 0, i_{1} \geq 0, i_{2} \geq 0\right\}
$$

which is a triangle and on its sides we have:

$$
\left\{\begin{array}{l}
s=0 \Longrightarrow s^{\prime}=b_{1}+\gamma_{1} i_{1}+\gamma_{2} i_{2} \\
i_{1}=0 \Longrightarrow i_{1}^{\prime}=p \lambda_{2} s i_{2} \\
i_{2}=0 \Longrightarrow i_{2}^{\prime}=q \lambda_{1} s i_{1}
\end{array}\right.
$$

It follows that $D$ is positively invariant and the disease free equilibrium $(1,0,0)$ is the only rest point on $\partial D$, the boundary of $D$. Indeed our vector field points inward on $\partial D-\{(1,0,0)\}$. So every solution of the system $(2-1)^{\prime}-(2-3)^{\prime}$ which starts in $\partial D-\{(1,0,0)\}$, immediately gets into $\stackrel{\circ}{D}$, the interior of $D$. From now on, we examine the dynamics of this system in the feasibility region $D$. The following theorem is a result of [3] concerning the nonexistence of certain types of solutions.

THEOREM 3.1. Let $f$ be a smooth vector field in $\mathbb{R}^{3}$ and $\gamma(t)$ be a closed piecewise smooth curve which is the boundary of an orientable smooth surface $S \subset \mathbb{R}^{3}$. Suppose $g: U \longrightarrow \mathbb{R}^{3}$ is defined and is smooth in a neighborhood $U$ of $S$. Moreover it satisfies $g(\gamma(t)) \cdot f(\gamma(t)) \geq 0$ and (curl $g) \cdot n<0$, where $n$ is the unit normal to $S$. Then $\gamma$ is not a finite union of the orbits of the system (2-5). 
In order to apply the above theorem, we define $g=g_{1}+g_{2}+g_{3}$ where

$$
\begin{gathered}
g_{1}\left(i_{1}, i_{2}\right)=\left[0,-\frac{f_{3}\left(i_{1}, i_{2}\right)}{i_{1} i_{2}}, \frac{f_{2}\left(i_{1}, i_{2}\right)}{i_{1} i_{2}}\right], \\
g_{2}\left(s, i_{2}\right)=\left[\frac{f_{3}\left(s, i_{2}\right)}{s i_{2}}, 0,-\frac{f_{1}\left(s, i_{2}\right)}{s i_{2}}\right], \\
g_{3}\left(s, i_{1}\right)=\left[-\frac{f_{2}\left(s, i_{1}\right)}{s i_{1}},-\frac{f_{1}\left(s, i_{1}\right)}{s i_{1}}, 0\right],
\end{gathered}
$$

and $f_{1}, f_{2}$ and $f_{3}$ denote the right hand side of $(2-1)^{\prime},(2-2)^{\prime}$ and $(2-3)^{\prime}$ reduced to functions of two variables by using $\sum=1$, respectively. Now after some computations $[2,6]$, we get

$$
(\operatorname{curl} g) \cdot(1,1,1)=-\left(\frac{p \lambda_{2}}{i_{1}^{2}}+\frac{q \lambda_{1}}{i_{2}^{2}}+\frac{b_{1}+\gamma_{1}}{i_{2} s^{2}}+\frac{b_{1}+\gamma_{2}}{i_{1} s^{2}}\right)
$$

Corollary 3.2. The system $(2-1)^{\prime}-(2-3)^{\prime}$ has no periodic orbits, homoclinic orbits or phase polygons in $\stackrel{\circ}{D}$.

Proof. We use Theorem 3.1 for $f=\left(f_{1}, f_{2}, f_{3}\right)$. Here we have $g . f=0$ and (curl $g) \cdot(1,1,1)<0$ in $\stackrel{\circ}{D}$.

LEMMA 3.3. The $\omega$-limit set of any orbit of the system $(2-1)^{\prime}-(2-3)^{\prime}$ with initial point in $D$ is a rest point.

Proof. Suppose the contrary, then the $\omega$-limit set has a regular point in $\stackrel{\circ}{D}$. Let $x$ be such a point and $h$ be its first return map. For a point $y$ near $x$ on the transversal, let $V$ be the region surrounded by the orbit $\gamma$ from $y$ to $h(y)$ and the segment between them. This region is known as Bendixson sack. (See Figure 1.)

Now by Stokes' theorem

$$
\iint_{V}(\operatorname{curl} g) \cdot(1,1,1) d \sigma=\int_{\gamma} g \cdot f d t+\int_{0}^{1} g(t y+(1-t) h(y)) \cdot(y-h(y)) d t .
$$

Since $g . f=0$ and $h(x)=x$, the right hand side of the above equality tends to zero as $y$ tends to $x$. But the left hand side tends to the integral over the region bounded by the $\omega$-limit set. This is a contradiction since $(\operatorname{curl} g) \cdot(1,1,1)<0$ in $\stackrel{\circ}{D}$.

REMARK 3.4. When the $\omega$-limit set lies in $\stackrel{\circ}{D}$, the above result is easily concluded by the generalized Poincaré-Bendixson theorem [18, 19] and Corollary 4.2. Similarly if the $\alpha$-limit set of an orbit of the system $(2-1)^{\prime}-(2-3)^{\prime}$ lies in $\stackrel{\circ}{D}$, it must be a single point.

4. The Planar System. Consider the proportions system $(2-1)^{\prime}-(2-3)^{\prime}$ in its feasibility region $D$. Using the equality $s+i_{1}+i_{2}=1$, we see that this system is essentially two dimensional. Thus we can eliminate one of the variables, say $s$, to arrive at the following quadratic planar system

$\begin{cases}i_{1}^{\prime}=\left(p \lambda_{1}-b-\varepsilon-\gamma_{1}\right) i_{1}+p \lambda_{2} i_{2}+\left(i_{1}+i_{2}\right)\left(\left(b_{2}+\varepsilon-p \lambda_{1}\right) i_{1}-p \lambda_{2} i_{2}\right), & (3-1) \\ i_{2}^{\prime}=q \lambda_{1} i_{1}+\left(q \lambda_{2}-b-\varepsilon-\gamma_{2}\right) i_{2}+\left(i_{1}+i_{2}\right)\left(\left(b_{2}+\varepsilon-q \lambda_{2}\right) i_{2}-q \lambda_{1} i_{1}\right) . & (3-2)\end{cases}$ 


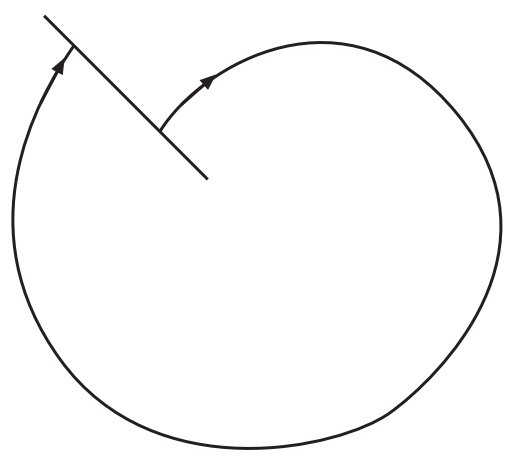

FIG. 3.1. The Bendixson sack.

The dynamics of the system $(2-1)^{\prime}-(2-3)^{\prime}$ on $D$ is equivalent to the dynamics of this planar system in the positively invariant region

$$
D_{1}=\left\{\left(i_{1}, i_{2}\right) \mid i_{1} \geq 0, i_{2} \geq 0, i_{1}+i_{2} \leq 1\right\} .
$$

The matrix of the linearization of the system $(3-1),(3-2)$ at the origin is:

$$
C=\left[\begin{array}{lc}
p \lambda_{1}-b-\varepsilon-\gamma_{1} & p \lambda_{2} \\
q \lambda_{1} & q \lambda_{2}-b-\varepsilon-\gamma_{2}
\end{array}\right],
$$

with $\operatorname{det} C=\left(b+\varepsilon+\gamma_{1}\right)\left(b+\varepsilon+\gamma_{2}\right)-p \lambda_{1}\left(b+\varepsilon+\gamma_{2}\right)-q \lambda_{1}\left(b+\varepsilon+\gamma_{1}\right)$. We set $R_{0}=\frac{p \lambda_{1}}{b+\varepsilon+\gamma_{1}}+\frac{q \lambda_{2}}{b+\varepsilon+\gamma_{2}}$. Hence if $R_{0}<1$, then $\operatorname{det} C>0$ and trace $C<0$ and if $R_{0}>1$ then $\operatorname{det} C<0$. Thus we have proved the following lemma.

LEMma 4.1. Let $R_{0}$ be the above threshold. Then the origin is a sink (resp. a saddle) for the system $(3-1),(3-2)$ whenever $R_{0}<1$ (resp. $R_{0}>1$ ).

Lemma 4.2. The trace of the linearization of the system $(3-1),(3-2)$ at a rest point in $\stackrel{\circ}{D}_{1}$ is negative.

Proof. We compute the trace at a rest point in $\stackrel{\circ}{D}_{1}$.

$$
\begin{aligned}
& \frac{\partial i_{1}^{\prime}}{\partial i_{\gamma}}=p \lambda_{1}-b-\varepsilon-\gamma_{1}+\left(b_{2}+\varepsilon-p \lambda_{1}-p \lambda_{2}\right) i_{2}+2\left(b_{2}+\varepsilon-p \lambda_{1}\right) i_{1}, \\
& \frac{\partial i_{2}}{\partial i_{2}}=q \lambda_{2}-b-\varepsilon-\gamma_{2}+\left(b_{2}+\varepsilon-q \lambda_{2}-q \lambda_{1}\right) i_{1}+2\left(b_{2}+\varepsilon-q \lambda_{2}\right) i_{2} .
\end{aligned}
$$

From $i_{1}^{\prime}=0$ and $i_{2}^{\prime}=0$, we get

$$
\begin{aligned}
& \frac{\partial i_{1}^{\prime}}{\partial i_{1}}=-p \lambda_{2} \frac{i_{2}}{i_{1}}+p \lambda_{2} \frac{i_{2}^{2}}{i_{1}}+\left(b_{2}+\varepsilon-p \lambda_{1}\right) i_{1}=-p \lambda_{2} \frac{i_{2}}{i_{1}}\left(1-i_{2}\right)+\left(b_{2}+\varepsilon-p \lambda_{1}\right) i_{1}, \\
& \frac{\partial i_{2}^{\prime}}{\partial i_{2}}=-q \lambda_{1} \frac{i_{1}}{i_{2}}+q \lambda_{1} \frac{i_{1}^{2}}{i_{2}}+\left(b_{2}+\varepsilon-q \lambda_{2}\right) i_{2}=-q \lambda_{1} \frac{i_{1}}{i_{2}}\left(1-i_{1}\right)+\left(b_{2}+\varepsilon-q \lambda_{2}\right) i_{2} .
\end{aligned}
$$

Using the equality $s+i_{1}+i_{2}=1$, we obtain

$$
\frac{\partial i_{1}^{\prime}}{\partial i_{1}}+\frac{\partial i_{2}^{\prime}}{\partial i_{2}}=\left(b_{2}+\varepsilon-\lambda_{1}\right) i_{1}+\left(b_{2}+\varepsilon-\lambda_{2}\right) i_{2}-p \lambda_{2} \frac{i_{2} s}{i_{1}}-q \lambda_{1} \frac{i_{1} s}{i_{2}} .
$$

Now from $(2-1)^{\prime}$ we have

$$
s^{\prime}=b_{1}\left(i_{1}+i_{2}\right)+b_{2} s\left(i_{1}+i_{2}\right)+\gamma_{1} i_{1}+\gamma_{2} i_{2}+\left(\varepsilon-\lambda_{1}\right) i_{1} s+\left(\varepsilon-\lambda_{2}\right) i_{2} s=0 .
$$


Thus $\left(b_{2}+\varepsilon-\lambda_{1}\right) i_{1} s+\left(b_{2}+\varepsilon-\lambda_{2}\right) i_{2} s<0$ and it follows that $\frac{\partial i_{1}^{\prime}}{\partial i_{1}}+\frac{\partial i_{2}^{\prime}}{\partial i_{2}}<0$.

Corollary 4.3. The system $(3-1),(3-2)$ has no source in $\stackrel{\circ}{D}_{1}$.

COROLlary 4.4. Every nondegenerate rest point of the system $(3-1),(3-2)$ in $\stackrel{\circ}{D}_{1}$ is hyperbolic.

The above two corollaries are immediate consequences of Lemma 4.2. Notice that a nondegenerate rest point of the system $(3-1),(3-2)$ is obtained by a transversal intersection of the two conic sections $i_{1}^{\prime}=0$ and $i_{2}^{\prime}=0$.

Proposition 4.5. There is at most one rest point in $\stackrel{\circ}{D}_{1}$ for the system $(3-$ $1),(3-2)$. Moreover such a rest point is always hyperbolic.

Proof. From the equilibrium conditions $i_{1}^{\prime}=i_{2}^{\prime}=0$, we get the following equation which is homogeneous with respect to $i_{1}$ and $i_{2}$ of second order.

$$
\begin{aligned}
& \left(\left(p \lambda_{1}-b-\varepsilon-\gamma_{1}\right) i_{1}+p \lambda_{2} i_{2}\right)\left(\left(b_{2}+\varepsilon-q \lambda_{2}\right) i_{2}-q \lambda_{1} i_{1}\right) \\
= & \left(q \lambda_{1} i_{1}+\left(q \lambda_{2}-b-\varepsilon-\gamma_{2}\right) i_{2}\right)\left(\left(b_{2}+\varepsilon-p \lambda_{1}\right) i_{1}-p \lambda_{2} i_{2}\right) .
\end{aligned}
$$

This equality can be written as

$$
q \lambda_{1}\left(b_{1}+\gamma_{1}\right) i_{1}^{2}+(*) i_{1} i_{2}-p \lambda_{2}\left(b_{1}+\gamma_{2}\right) i_{2}^{2}=0
$$

where $(*)$ is a statement in terms of the involved parameters. The set of all roots of this quadratic equation consists of two lines through the origin in the $\left(i_{1}, i_{2}\right)$ plane. One of these lines has negative slope and meets $D_{1}$ only at the origin. Thus the other line contains all rest points of the system $(3-1),(3-2)$ in $D_{1}$. Since each line contains at most two rest points of a quadratic planar system and this line contains the origin, it follows that $\stackrel{\circ}{D}_{1}$ contains at most one rest point. This rest point is obtained by a transversal intersection of this line and each of the conic sections $i_{1}^{\prime}=0$ or $i_{2}^{\prime}=0$. It is easy to see that at this rest point, these two conic sections intersect transversally. Therefore this rest point is nondegenerate and by Corollary 4.4, it must be hyperbolic.

In the above argument, we have indeed shown that all rest points of the system $(3-1),(3-2)$ which are not more than three points, are nondegenerate, except the origin in the case $R_{0}=1$. Now we are ready to prove our main result concerning the dynamics of the system $(2-1)^{\prime}-(2-3)^{\prime}$ in $D$.

THEOREM 4.6. (i) If $R_{0} \leq 1$, then $(1,0,0)$ is a global attractor in $D$.

(ii) If $R_{0}>1$, then there exists a unique rest point (an endemic equilibrium) in $\stackrel{\circ}{D}$ which attracts $D-\{(1,0,0)\}$.

Proof. When $R_{0}<1$, the origin is a sink for the planar system $(3-1),(3-2)$. If there exists another rest point in $\stackrel{\circ}{D}_{1}$ for this system, it must be unique and hyperbolic. By Corollary 4.3, it cannot be a source. If it is a sink, then we will have two sinks in $D_{1}$. The basins of attraction of these two points are open and by Lemma 3.3, $D_{1}$ is the union of these two open subsets. This contradicts the connectedness of $D_{1}$ and shows that it cannot be a sink. Now suppose that there is a saddle point in $\stackrel{\circ}{D_{1}}$. By Corollary 3.2 , there is no homoclinic orbit in $\stackrel{\circ}{D_{1}}$. Hence the origin attracts the unstable manifold of the saddle point. Now the region bounded by the unstable manifold contains some part of the stable manifold of of the saddle point. Thus the 
$\alpha$-limit set of this part of the stable manifold is a rest point in $D_{1}$ by Remark 3.4. This is a contradiction since this rest point can be neither the origin nor the saddle point. Therefore the origin is the only rest point in $D_{1}$ for the system $(3-1),(3-2)$ and by Lemma 2.3, it is the $\omega$-limit set of all points of $D_{1}$.

The above fact is still valid for the limiting case, $R_{0}=1$. To see this, suppose that there exists another rest point in $D_{1}$, then it must be hyperbolic and belong to $\stackrel{\circ}{D}_{1}$. Thus it remains in $\stackrel{\circ}{D}_{1}$ when the involved parameters are slightly changed to get $R_{0}<1$ which contradicts the above result. This finishes the proof of (i).

Now suppose $R_{0}>1$. Then the origin is a saddle point for the planar system $(3-1),(3-2)$. Thus by Lemma 2.3 , there must be some rest point in $\stackrel{\circ}{D}_{1}$. Since such a rest point is unique and hyperbolic, it must be a sink and attract all points of $D_{1}$ except the stable manifold of the origin. We claim that the stable manifold meets $D_{1}$ only at the origin. To see this notice that some part of the unstable manifold of the origin must be outside of $D_{1}$ for its right angle. Since $D_{1}$ is positively invariant, the stable manifold does not intersect $\stackrel{\circ}{D}_{1}$. Moreover, the vector field points inward on $\partial D_{1}-\{(0,0)\}$. Thus the stable manifold of the origin does not intersect $\partial D_{1}-\{(0,0)\}$ either. This shows that the origin cannot attract any point of $D_{1}-\{(0,0)\}$. Thus the unique sink attracts $\stackrel{\circ}{D}_{1}-\{(0,0)\}$. It means that there is a unique rest point in $\stackrel{\circ}{D}$ for the system $(2-1)^{\prime}-(2-3)^{\prime}$ which attracts $D-\{(1,0,0)\}$.

REMARK 4.7. In the above argument, in order to prove the global asymptotic stability of the endemic equilibrium (i.e. the unique rest point in $\stackrel{\circ}{D}_{1}$ ), we showed that the stable manifold of the origin cannot intersect $\stackrel{\circ}{D}_{1}$. It is a special case of the following fact. Let $X$ be a smooth vector field on a smooth manifold $M$ and $D \subset M$ is a positively invariant region with a saddle point on $\partial D$. If the unstable manifold of this saddle point contains a point of $(M-D)^{\circ}$, then its stable manifold cannot intersect $\stackrel{\circ}{D}$. In order to prove it, one can follow our proof in the special case and observe that this is a direct consequence of the Hartman-Grobman theorem. However, it is obvious by the Inclination Lemma [18].

Conclusion. The threshold $R_{0}=\frac{p \lambda_{1}}{b+\varepsilon+\gamma_{1}}+\frac{q \lambda_{2}}{b+\varepsilon+\gamma_{2}}$ clearly shows the effect of each group on the epidemics process. In the differential infectivity model, we have a group of superspreaders, say $I_{2}$ with $\lambda_{2} \gg \lambda_{1}$. This group may be very small with $q \ll 1$, but the fact $\lambda_{2} \gg \lambda_{1}$ causes the term $q \lambda_{2}$ to be significant in $R_{0}$ and it can force $R_{0}$ to be greater than one. In most of applications, the initial conditions of the population is nearby the disease free equilibrium and in almost all epidemiological models, the behavior of the system is known around the disease free equilibrium. In our model, this equilibrium is unstable when $R_{0}>1$ and our state which is close to the disease free equilibrium initially, gets far from it and tends to the endemic equilibrium. This proves the assertion of [11] that the disease is primarily spread by a small, highly infectious, group of superspreaders.

Now suppose that $I_{2}$ is the post-infection core group. Then we have again a small group with $q \ll 1$ and $\lambda_{2} \gg \lambda_{1}$. Similar to the above argument, this group also plays a crucial role in the epidemic process. Now our analysis shows that psychological parameters are also very important, yet often neglected, factors in the spread of disease transmission. In the differential infectivity model, $q$ is a biological parameter and difficult to control, but in this case, it is a psychological parameter and there are well-known methods in social psychology to reduce it. 
Acknowledgments. The author would like to thank Institute for Studies in Theoretical Physics and Mathematics for supporting this research. I also wish to thank the referees for their invaluable suggestions.

\section{REFERENCES}

[1] Busenberg, S., VAn Den Driessche, P., Analysis of a disease transmission model in a population with varying size, J. Math. Bio., 28 (1990), pp. 257-270.

[2] Busenberg, S., VAn Den Driessche, P., Nonexistence of periodic solutions for a class of epidemiological models. In: Busenberg, S., Martelli, M., (eds.), Differential Equations Models in Biology, Epidemiology and Ecology. (Lecture Notes in Biomathematics, Vol. 92), Springer-Verlag, Berlin, Heidelberg, 1991.

[3] Busenberg, S., van den Driessche, P., A method for proving the nonexistence of limit cycles, J. Math. Anal. Appl., 172 (1993), pp. 463-479

[4] Capasso, V., Mathematical structure of the epidemic systems, Lecture Notes in Biomathematics vol. 97, Springer-Verlag, Berlin, Heidelberg, 1993.

[5] Castillo-Chavez, C., Cooke, K.L., Huang, W., Levin, S.A., On the role of long incubation periods in the dynamics of acquired immunodeficiency syndrome (AIDS). Part 2: Multiple group models. In: Castillo-Chavez, C. (ed.), Mathematical and Statistical Approaches to AIDS Epidemiology. (Lecture Notes in Biomathematics, Vol. 83), Springer-Verlag, Heidlberg, 1989.

[6] Derrick W.R., VAn den Driessche, P., A disease transmission model in a nonconstant population, J. Math. Bio., 31 (1993), pp. 495-512.

[7] Hethcote, H.W., VAN ARK, J.W., Epidemiological models for heterogeneous populations: proportionate mixing, parameter estimation and immunization programs, Math. Biosci., 84 (1987), pp. 85-118.

[8] Hethcote, H.W., Yorke, J.A., Gonorrhea Transmission Dynamics and Control, (Lecture Notes in Biomathematics, vol. 56), Springer-Verlag, Berlin, Heidelberg, 1984.

[9] Huang, W., Cooke, K.L., Castillo-Chavez, C., Stability and bifurcation for a multiple-group model for the dynamics of HIV/AIDS transmission, SIAM J. Appl. Math., 52 (1992), pp. $835-854$.

[10] Hyman, J.M., Li, J., Stanley, E.A., The differential infectivity and the staged progression model for the transmission of HIV, Math. Biosci., 155 (1999), pp. 77-109.

[11] Hyman, J.M., Li, J., Stanley, E.A., The initialization and sensitivity of multigroup models for the transmission of HIV, J. Theor. Bio., 208 (2001), pp. 227-249.

[12] Kribs-Zaleta, C.M., Structured models for heterosexual disease transmission, Math. Biosci., 160 (1999), pp. 83-108.

[13] Kribs-Zaleta, C.M., Core recruitment effects in SIS models with constant total populations, Math. Biosci., 160 (1999), pp. 109-158.

[14] Lajmanovich, A., Yorke, J.A., A deterministic model for gonorrhea in a nonhomogeneous population, Math. Biosci., 28 (1976), pp. 221-236.

[15] May, R.M., Anderson, R.M., MCLean, A.R., Possible demographic consequences of $H I V / A I D S$ epidemics I. Assuming HIV infection always leads to AIDS, Math. Biosci., 90 (1988), pp. $475-505$.

[16] May, R.M., Anderson, R.M., McLean, A.R., Possible demographic consequences of $H I V / A I D S$ epidemics II. Assuming HIV infection does not necessarily lead to AIDS. In Castillo-Chavez, C., Levin, S.A., Shoemaker, C.A. (eds.), Mathematical Approaches to Problem in Resource Management and Epidemiology, (Lecture Notes in Biomathematics, vol. 81), Springer-Verlag, Berlin, Heidelberg, 1989.

[17] Nold, A., Heterogeneity in disease transmission, Math. Biosci., 52 (1980), pp. 227-240.

[18] Palis, J., DeMelo, W., Geometric Theory of Dynamical Systems, An introduction, SpringerVerlag, New York, Heidelberg, Berlin, 1982.

[19] Perko, L., Differential Equations and Dynamical Systems, (Third edition), Springer-Verlag, New York, Inc., 2001.

[20] Sattenspiel. L., The structure and social interactions and the spread of HIV. In: CastilloChavez, C.(ed.), Mathematical and statistical Approaches to AIDS Epidemilogy ,(Lecture Notes in Biomathematics, vol. 83), Springer-Verlag, Heidelberg, 1989. 